\title{
Infrared Spectra of Brown Dwarf Candidates in Taurus
}

\author{
A. Magazzù \\ INAF-Centro Galileo Galilei, Apartado 565, 38700 S/C de La Palma, \\ Spain \\ C. Dougados
}

Laboratoire d'Astrophysique de l'Observatoire de Grenoble, 414 rue de la Piscine, 38041 Grenoble, France

J. Licandro

Isaac Newton Group of Telescopes, Apartado 321, $38700 S / C$ de La Palma, Spain

E. L. Martín

Institute for Astronomy, University of Hawaii, 2680 Woodlawn Drive, Honolulu, HI 96822

E. A. Magnier, F. Ménard

Canada-France-Hawaii Telescope Corporation, P.O. Box 1597, Kamuela, HI 96743

\begin{abstract}
We have obtained low resolution infrared spectra of 21 brown dwarf candidates, selected from a survey performed at the Canada-FranceHawaii Telescope. The spectra were obtained at the Telescopio Nazionale Galileo (La Palma), using the NICS spectrometer in the Amici configuration, which allows to observe the range $0.85-2.45 \mu \mathrm{m}$ in a single shot. The bands shown by most of the spectra confirm that we are dealing with low effective temperature objects. Preliminary results of spectral classification are presented.
\end{abstract}

\section{Introduction}

Last year, Martín et al. (2001) identified four brown dwarfs (BDs) in the Taurus star forming region (SFR), by means of optical low resolution spectroscopy of candidates selected from photometry obtained at Canada-France-Hawaii Telescope (details in Dougados et al. 2002, in preparation). Martín et al.'s results suggest that the density of BDs in Taurus might be anti-correlated with the density of T Tauri stars. This could explain the lack of BDs in the searches carried out by Briceño et al. (1998) and Luhman et al. (2000). A larger sample of BDs is required in order to answer this and other open issues. Here we present infrared spectra of 16 new BDs candidates and 5 previous known BDs 


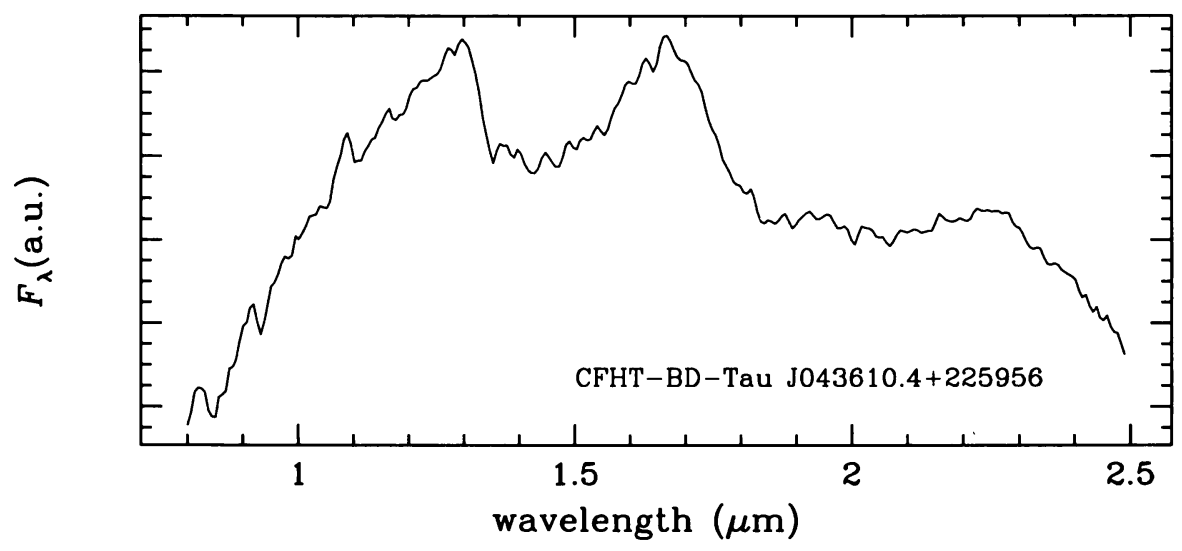

Figure 1. Spectrum of CFHT-BD-Tau J043610.4+225956

in the Taurus SFR. The advantage of our low resolution IR spectroscopy is the possibility to reach fainter candidates with little time effort, and to have the whole $0.85-2.45 \mu \mathrm{m}$ range in a single spectrum.

\section{Observations and Results}

Observations were performed on November 2001 with the Italian Telescopio Nazionale Galileo, in La Palma, Canary Islands. The Near Infrared Camera and Spectrograph (NICS, Baffa et al. 2001) was used in the Amici mode, which gives a low resolution (resolving power $\sim 50$ ) long-slit spectrum covering the range $0.85-2.45 \mu \mathrm{m}$ (Oliva 2001). Magnitudes of our sources range from $J \sim 13$ to $J \sim 16$. Typical exposure times varied from 2 to 15 minutes, depending on source brightness. Wavelength calibration was performed using an argon lamp and telluric features. Each spectrum was divided by an A0 reference star spectrum, and normalized using a synthetic A0 spectrum smoothed to the appropriate resolution. As an example of the obtained spectra, in Figure 1 we show the spectrum of CFHT-BD-Tau J043610.4+225956, classified as brown dwarf from optical spectroscopy by Martín et al. (2001),

A preliminary determination of spectral types was made using indices from Lucas et al. (2001). These indices are defined as $W=F_{\nu}(1.50 \mu \mathrm{m}) / F_{\nu}(1.682 \mu \mathrm{m})$ and $Q=(F 1 / F 2) /(F 3 / F 2)^{1.219}$ (reddening-independent index), where $F 1$ is the $F_{\nu}$ flux at $1.57 \mu \mathrm{m}, F 2$ is $F_{\nu}$ flux at $1.682 \mu \mathrm{m}$, and $F 3$ is the $F_{\nu}$ flux at $1.79 \mu \mathrm{m}$. In Table 1 we show the indices $W, Q$, and the spectral type obtained from these indices after a critical comparison with templates from Testi et al. (2001). A rough estimate of reddening effects has been obtained by using the empirical selective extinction function of Cardelli, Clayton, \& Mathis (1989).

Following Martín et al. (2001), we assume that a brown dwarf the in Taurus SFR will have spectral type M7 or later. Excluding the already known objects, we remain with six targets which satisfy this condition and thus can 
Table 1. Indices and Spectral types

\begin{tabular}{lccc}
\hline Name & $\mathrm{Q}$ & $\mathrm{W}$ & Sp.Type \\
\hline CFHT-BD-Tau J041308.8+280556 & 0.70 & 0.79 & $\mathrm{M} 4$ \\
CFHT-BD-Tau J041419.4+275701 & 0.74 & 0.76 & $\mathrm{M} 4$ \\
CFHT-BD-Tau J041514.7+280010 & 0.54 & 0.66 & $\mathrm{~L} 1$ \\
CFHT-BD-Tau J041817.2+282842 & 0.66 & 0.61 & $\mathrm{M} 6$ \\
CFHT-BD-Tau J041802.2+281750 & 0.91 & 0.69 & $\mathrm{M} 4$ \\
CFHT-BD-Tau J041851.2+281434 & 0.63 & 0.68 & $\mathrm{M} 7$ \\
CFHT-BD-Tau J043406.2+241851 & 0.62 & 0.68 & $\mathrm{M} 8$ \\
CFHT-BD-Tau J043414.3+241606 & 0.72 & 0.72 & $\mathrm{M} 4$ \\
CFHT-BD-Tau J043453.7+241052 & 0.57 & 0.74 & $\mathrm{M} 6$ \\
CFHT-BD-Tau J043208.6+242212 & 0.77 & 0.68 & $\mathrm{M} 4$ \\
CFHT-BD-Tau J043221.6+241121 & 0.56 & 0.72 & $\mathrm{M} 7$ \\
CFHT-BD-Tau J043415.2+225032 & 0.60 & 0.63 & $\mathrm{M} 7$ \\
CFHT-BD-Tau J043610.4+225956 & 0.56 & 0.63 & $\mathrm{M} 8$ \\
CFHT-BD-Tau J043551.4+224911 & 0.55 & 0.60 & $\mathrm{~L} 0$ \\
CFHT-BD-Tau J043638.9+225813 & 0.53 & 0.62 & $\mathrm{~L} 0$ \\
CFHT-BD-Tau J043627.6+223950 & 0.62 & 0.75 & M8 \\
CFHT-BD-Tau J043248.1+243108 & 0.57 & 0.63 & L0 \\
CFHT-BD-Tau J044306.3+252324 & 0.81 & 0.76 & M4 \\
CFHT-BD-Tau J044229.7+251948 & 0.68 & 0.56 & M5 \\
CFHT-BD-Tau J044001.7+255630 & 0.70 & 0.71 & M4 \\
CFHT-BD-Tau J044110.7+255512 & 0.71 & 0.67 & M5 \\
\hline \hline
\end{tabular}

be considered as very likely substellar members of Taurus SFR. These objects are reported in Table 2 (infrared magnitudes are from the Two Micron All Sky Survey second incremental catalog).

Table 2. New likely substellar objects in Taurus

\begin{tabular}{lcccc}
\hline Name & $\mathrm{J}$ & $\mathrm{H}$ & $\mathrm{K}$ & Sp.Type \\
\hline CFHT-BD-Tau J041514.7+280010 & 15.095 & 14.257 & 13.743 & L1 \\
CFHT-BD-Tau J043406.2+241851 & 14.074 & 13.474 & 13.053 & M8 \\
CFHT-BD-Tau J043221.6+241121 & 15.865 & 15.381 & 14.759 & M7 \\
CFHT-BD-Tau J043551.4+224911 & 15.493 & 14.670 & 14.173 & L0 \\
CFHT-BD-Tau J043627.6+223950 & 15.718 & 15.143 & 14.771 & M8 \\
CFHT-BD-Tau J043248.1+243108 & 15.997 & 15.432 & 14.689 & L0 \\
\hline \hline
\end{tabular}

An accurate determination of IR spectral types in a SFR implies a careful study of reddening and gravity effects. This can give also information on the membership of our candidates, and thus can provide a definitive assessment of their substellar status. We are presently working with a more complete set of templates and synthetic spectra. The results of this analysis will be presented elsewhere (Magazzù et al. 2002, work in progress).

Support for this work was provided by the National Aeronautics and Space Administration (NASA) grant NAG5-9992 and National Science Foundation (NSF) grant AST-0205862. 


\section{References}

Baffa, C., et al. 2001, A\&A, 378, 722

Briceño, C., Hartmann, L., Stauffer, J.R., \& Martín, E.L. 1998, AJ, 115, 2074

Cardelli, J.A., Clayton, G.C., \& Mathis, J.S. 1989, ApJ, 345, 245

Martín, E.L., Dougados, C., Magnier, E., Ménard, F., Magazzù, A., Cuillandre, J.-C., \& Delfosse, X. 2001, ApJ, 561, L195

Lucas, P.W., Roche, P.F., Allard, F., \& Hauschildt, P.H. 2001, MNRAS, 326, 695

Luhman, K.L., Briceño, C., Rieke, G.H., Hartmann, L. 1998, ApJ, 493, 909

Oliva, E. 2001, Mem. Soc. Astr. It., 71, 861

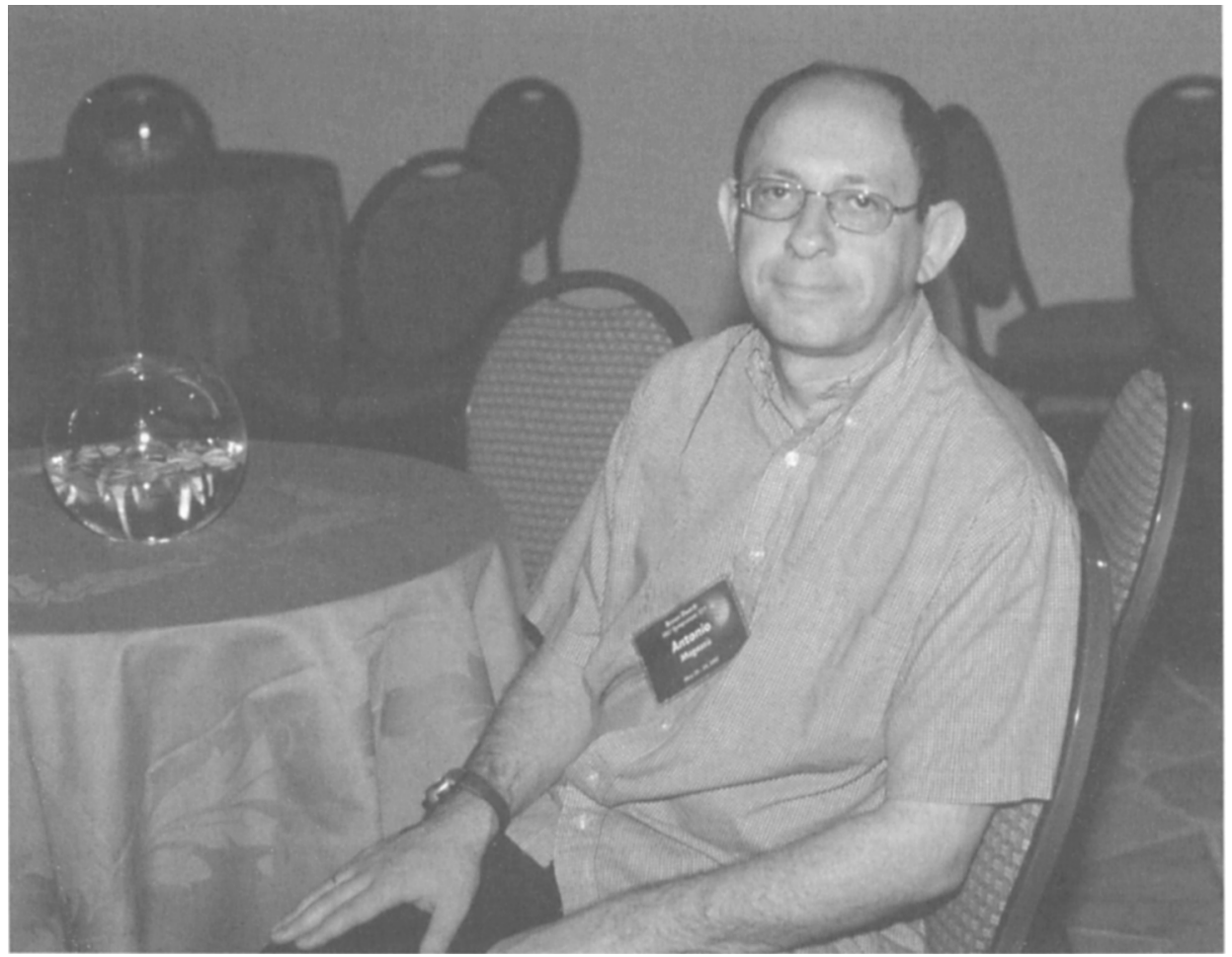

Antonio Magazzù 